\title{
Surrogate beta cells
}

\author{
S. Ferber ${ }^{1}$, H . H eimberg ${ }^{2}$, M. B rownlee 3 , C. C olton ${ }^{4}$ \\ ${ }^{1}$ Institute of Endocrinology, Sheba Medical Center, Tell-Hashomer, Israel \\ ${ }^{2}$ Department of Cell Biology and Physiology, Washington University School of Medicine, St. Louis, Missouri, USA \\ ${ }^{3}$ Diabetes Research Center, Albert Einstein College of Medicine, New York, USA \\ ${ }^{4}$ Department of Chemical Engineering, Massachusetts Institute of Technology, Boston, Massachusetts, USA
}

Experiments with diabetic animals and patients (most notably the Diabetes Control and Complications Trial) have proven that normalization of blood glucose will prevent the onset and progress of diabetic complications. Islet cell transplantation seems the obvious way to achieve normalization of blood glucose in patients with both insulin-dependent (IDDM) and non-insulin-dependent (NIDDM) diabetes mellitus, but there are several major obstacles to be overcome. The obstacles fall into two broad categories. First is the category of immunologic rejection. Since the adverse effects of non-selective immunosupressive regimens are unacceptable for an otherwise healthy diabetic patient, solutions must be found that specifically abrogate transplant rejection. If human cells are the donor source, this means overcoming the allorejection mechanism, which is currently thought to be mediated primarily by CD 8 + cytotoxic lymphocytes which recognize specific antigens associated with beta-cell class I MHC molecules, "dock" with these targets, and stimulate destruction of the graft cells. If animal cells are the donor source, this means overcoming the mechanisms of xenorejection, which involve an immediate, hyperacute rejection phase that destroys the graft in hours, caused by preexisting xenoreactive natural antibodies that bind primarily to a single saccharide epitope ( $\mathrm{Gal}$ alpha $1-3 \mathrm{Gal}$ ) which is present on most animal tissues but is absent in man. Binding of antibody activates

Participants: A. Hayek, The Whittier Institute, La Jolla, California, USA

C. Newgard, University of Texas Southwestern Center for Diabetes Research, Dallas, Texas, USA

S. Efrat, Department of Molecular Pharmacology, Albert Einstein College of Medicine, Bronx, New York, USA

Corresponding author: M. Brownlee, M.D., Diabetes Research Center, Albert Einstein College of Medicine, 1300 Morris Park Avenue, Bronx, NY 10461, USA complement proteins in the patient's blood which bind to and destroy the graft. After the hyperacute phase of xenorejection, there is a chronic cell-mediated phase that appears to involve CD4 + effector cells producing low molecular weight toxic products such as cytokines and reactive oxygen species. Although specific inhibition of either allograft rejection or xenograft rejection would allow cure of non-autoimmune NIDDM, it is less certain that this would be sufficient for curing autoimmune IDDM. In IDDM patients, the problem of recurrent autoimmune destruction will also have to be addressed.

Over the past few years, exciting advances have been made towards the solution of these problems. Approaches to rejection and autoimmune destruction aim either 1) to modify the recipient's immune system, or 2) to modify the donor graft tissue. Approaches to modifying the recipient's immune system include a) creation of full or mixed chimerism, using temporary immunosupression plus donor bone marrow; b) implantation of islets into the thymus with transient immunsupression; and c) systemic administration of co-stimulator antagonists, which block the allogeneic T-cell activation that is dependent on ligation of T-cell CD28 with APC B7, and/or CD40 with gp39. Approaches to modifying donor graft tissue include for allorejection a) genetic engineering of graft tissue to express fas ligand [fasL], which lyses fasbearing activated $\mathrm{T}$ cells on contact: and $\mathrm{b}$ ) down regulation of MHC class I by genetic engineering; for the hyperacute phase of xenorejection, a) the expression of the human complement inhibitors daf and cd59 on transgenic organs; and b) the expression in transgenic organs of a competitive inhibitor of Gal alpha1-3 Gal, fucosyltransferase. For the cellular phase of xenorejection, and perhaps for recurrent autoimmunity as well, antiapoptosis genes such as bcl-2 have been shown to protect beta cells against cytokine-induced cell death. Lastly, immunoisolation of graft tissue 
has been proposed as a solution for allogeneic rejection, recurrent autoimmunity, and xenorejection.

After the problems of immunological rejection have been solved, the second category of obstacle that must be overcome before islet or beta-cell transplantation can be used clinically to cure all types of diabetes is that of donor tissue supply. Currently, the clinical demand for human islet cells far exceeds the supply, and even if animal sources were feasible, important issues of uniformity and absence of animal infectious agents require novel solutions. Three approaches where important progress has been made are a) the isolation and growth of islet precursor cells; b) genetic engineering of established beta-cell lines to optimize endocrine function; and c) the conditional imortalization of islet cells through genetic engineering.

The papers discussed in this work group present the state of the art in the general approaches described above for modification of donor graft tissue. They include the topics of Stem Cells and Islet Progenitors by Alberto Hayek, Engineered Beta-Cell Lines by Christopher Newgard, Transgenic Cell Lines by Shimon Efrat, and an overview of Immunoisolation of Islet Cells by Clark Colton.

\section{Alberto Hayek: Stem cells and islet progenitors}

Dr. Alberto Hayek discussed identification, isolation and characterization of stem cells and/or endocrine cell precursors from the human pancreas that could be used not only for the understanding of basic islet developmental issues but for fetal cell transplantation research. Dr. Hayek's long term goal is to ex vivo expand fetal pancreatic endocrine cells and to implant this tissue in order to correct insulin deficient states. Enzymatic digestion and culture of human fetal pancreas generates cell aggregates or clusters rich in endocrine precursor cells (islet-like cluster cells [ICC]). The endocrine cell content of the clusters is between 10 and $15 \%$; the rest are mostly undifferentiated epithelial cells. Extracellular matrix, produced and secreted by mouse bladder carcinoma cell lines and hepatic growth factor (HGF/SF) have additive effects on proliferation of human fetal pancreatic cells. Up to a 200-fold increase in cell number was achieved in these studies, at the cost of decreased human insulin content, which is a part of a dedifferentiation process the cells go through with the increased proliferation rate. A differentiation process occurs upon implantation in vivo. The insulin levels produced by ex vivo proliferated ICC were comparable to those produced by the same number of mature islets, after several weeks of implantation in nude mice. The renal capsule was found to be the optimal site for implantation in athymic nude mice [1]. Implantation of ICCs attached to agarose beads in the pancreas also gave good results, while implantation of naked ICCs in the liver or spleen did not result in substantial insulin output. Maturation and differentiation of ICC in the in vitro environment was partially achieved with nicotinamide (NIC), a potent inducer of endocrine differentiation in cultured human fetal pancreatic cells [2]. A potentiation of insulin gene expression occurred in cell aggregates but not in monolayers.

The pancreatic islet derives from the ductal epithelium. The evidence for this assumption is based on cell lineage analysis, transgenic mice expressing the interferon- $\gamma$ gene in pancreatic islets [3] and tissue recombination studies using adult epithelium and fetal mesenchyme. Several putative markers of ductal endocrine precursor cells have been published. They include the enzymes tyrosine hydroxylase, carbonic anhydrase II, $\beta$-galactosidase and glutamic acid decarboxylase (GAD); the cytokeratins, peptide hormones such as peptide YY and neuropeptide Y [2], the intermediate filament vimentin the antiapoptotic gene product of the bcl-2 gene and the newly found transcription factor PDX-1. An alternative hypothesis to help localize stem cells is based on the regulation of expression of membrane protein members of the different families of cell adhesion molecules. For some of the above named proteins, the absence of a clear precursor role will rule them out as true stem cell markers. This is probably the case for PDX-1 since mice carrying a null mutation of the homeobox gene lack a pancreas but in the early embryo glucagon and insulin cells develop. Dr. Hayek's laboratory has investigated the role of three different proteins as potential markers of endocrine precursor cells: GAD, $\beta$ galactosidase and the KSA antigen, renamed epithelial-cell adhesion molecule (Ep-CAM).

The $\beta$-galactosidase level in adult islets is significantly lower than in the fetal cell clusters. Interestingly, the enzyme preferentially localizes in some specific cells within the pancreatic ducts in the fetal pancreas and is also present in newly formed islets. Cell sorting experiments using a lipophilic fluorogenic $\beta$-galactosidase substrate produced populations enriched in precursor cells but the sorting did not completely exclude all differentiated endocrine cells. Nevertheless, the ability to sort viable cells offers an opportunity to isolate cells with the potential to become endocrine.

Ep-CAM exhibits several features of a morphoregulatory molecule involved in the development of pancreatic islets. Using quantitative conical immunofluorescence, Dr. Hayek's laboratory is showing very high levels of expression in cell clusters budding out of fetal pancreatic ducts with a reverse pattern of expression in the adult pancreas, high levels in ducts and low levels in islets. Ep-CAM expression was targeted to the lateral domain of epithelial cells in the pancreas and mediated calcium-independent cellcell interactions. In vitro, cell expansion experiments demonstrated up regulation of Ep-CAM expression while conditions that favoured the differentiation 
into endocrine cells, such as the addition of nicotinamide, caused the opposite effect. Thus, Ep-CAM is an example of how the developmentally regulated expression of an adhesion molecule may play a role in islet neogenesis. Upon identification of the correct markers to identify pancreatic stem cells, these advances in in vitro proliferation and expansion could significantly increase the supply of human islet cells available for allotransplantation of diabetic patients.

\section{Christopher B. Newgard: Engineered beta cell lines}

Dr. Christopher B. Newgard is taking a different approach to generate an unlimited supply of pancreatic islet cells for transplantation, and that is by modification of pre-existing beta-cell lines for enhanced function, using the tools of molecular biology. In designing such an approach, he has targeted the parameters that are desirable in surrogate beta cells, such that they will faithfully mimic normal pancreatic islet function. First, high levels of human insulin content and secretory capacity will allow the size of a transplanted graft be restrained to reasonable limits. Second, it is important that the engineered lines secrete insulin mainly by the so-called "regulated pathway", as opposed to constitutive release. The regulated pathway encompasses both acute regulation of insulin secretion and the complete processing of proinsulin to the mature insulin polypeptide. The presence of secretory granules and retention of proinsulin processing capacity represent a major advantage of insulinoma and other neuroendocrine cell lines over non-endocrine cell lines such as fibroblasts. A third, and certainly central parameter is to equip the cells with a capacity for normal glucose sensing. Glucose responsiveness has several components that must be considered, including the appropriate threshold for the response, rapid response dynamics, and the correct magnitude of response.

It is imperative that engineered cell lines retain phenotypic and genotypic stability, both in vitro and when implanted into animals or humans. This includes both genes that are inserted or deleted during the course of engineering and key endogenous genes. In the absence of this kind of stability, engineered cells will have no tangible advantage relative to isolated islets, even though procurement of islets is both costly and difficult. Further, it is anticipated that insulin producing cell lines will be transplanted in the context of perm-selective membranes or devices in order to protect the graft from the host immune system, and also to contain the transformed cell lines. The engineered lines must therefore grow and thrive in encapsulation devices, either naturally or as a result of further manipulation of the cells or membrane materials. A major potential advantage of cell lines relative to islets is that they can be grown in theoretically unlimited quantity at relatively low cost. This advantage can only be realized, however, with the development of methods for scale-up of cell growth from petri dishes to bioreactors. Finally, it will be useful to retain the capacity for further cell engineering, even after development of cells with the aforementioned properties, in case the encapsulation procedures chosen are incapable of fully protecting the implant from the immune system.

In an effort to achieve the above characteristics, the Newgard laboratory has developed a strategy for the step-wise stable introduction of genes relevant to beta-cell performance, a process that they term "iterative engineering". Rodent insulinoma cell lines engineered to contain multiple copies of the human insulin gene exhibit an increase in insulin content of more than 10-fold relative to untransfected cells, and process human proinsulin to mature insulin with high efficiency [4]. Cell lines that are further engineered to express the GLUT2 and glucokinase genes demonstrate stable expression of the three transgenes for the full lifetime of the lines produced to date (6 months to 1.5 years in continuous culture). The multiple engineered lines increase insulin secretion by $6-8$ fold in response to glucose, effects that are further potentiated in a fashion similar to that seen in beta cells by agents that raise cAMP. These responses are clearly larger than observed in unengineered cells, and perifusion studies reveal that insulin secretion is sustained in glucokinase and/or GLUT2 expressing cells more effectively than in unengineered cells or cells that express the human insulin transgene alone [3]. The engineered cell lines exhibit a maximal response to glucose at low concentrations $(0.05-0.25 \mathrm{mmol} / \mathrm{l})$, but can be shifted such that their maximal response occurs at 3-5 mmol/l glucose by performing secretion studies in the presence of $2 \mathrm{mmol} / \mathrm{l}$ 5-thioglucose, which inhibits low $\mathrm{K}_{\mathrm{m}}$ hexokinase activity and glucose usage by approximately $30 \%$ in these cells. These data suggest that further adjustment of glucose sensing to simulate the response threshold of the beta cell (4-5 mmol/l) should be achievable by stable suppression of low $\mathrm{K}_{\mathrm{m}}$ hexokinase activity. Dr. Newgard is currently investigating several strategies for achieving this goal, including antisense and gene "knock-out" strategies. Transplantation of the engineered RIN 1046-38 cell lines into nude rats reveals that stably integrated transgenes are expressed at constant levels in the in vivo environment over the full duration of experiments performed to date (48 days). Several endogenous genes expressed in normal beta cells, including rat insulin, amylin, sulfonylurea receptor, and glucokinase are stably expressed in the insulinoma lines during these in vivo studies. Endogenous GLUT2 expression, in contrast, is rapidly extinguished within 10 days of cell implantation. The loss of GLUT2 expression is overcome in engineered cell lines in which GLUT2 expression is provided by a stably transfected transgene. These results suggest that a potential 
advantage of the iterative engineering approach may be to preserve stability of function and phenotype, particularly in the in vivo setting. These results provide encouragement for upcoming studies on insulin replacement with engineered cells in animal models with chemically induced or spontaneous autoimmune diabetes. The rat insulinoma cell line RIN1046-38 was chosen to be used for the iterative engineering because it maintains a stable diploid state, it expresses only one insulin gene, the rat insulin 1 gene, and does not express glucagon or somatostatin. In an attempt to equip the cells with the ability to survive attacks by cytokines, the group has overexpressed the Mn-superoxide dismutase (Mn-SOD) gene in the engineered cells so that the enzyme will block the harmful effect of free radical formation. A tremendous effort has been devoted to engineering rodent cell lines to meet the criteria stated by Dr. Newgard, and much more still has to be done. An immediate focus will be expression of human insulin in rodent insulinoma cells and eliminating expression of the endogenous insulin gene(s), since rodent insulin is much more immunogenic and perhaps more mitogenic as well. This is necessitated by the lack of a suitable human insulinoma cell line for study, although several groups are currently attempting to produce such lines from human islet tissue. It is clear that the recent intense effort in cell design by genetic engineering will be followed by an equally active period of animal experimentation, wherein the formidable challenges of device design and immunological barriers to transplantation will be addressed. It is in this phase that the therapeutic applicability of this technology will be defined.

\section{Shimon Efrat: Transgenic cell lines}

Having the same ultimate goal in mind, namely the creation of an unlimited source of xenogeneic donor beta cells with well defined and reproducible characteristics, Dr. Shimon Efrat transformed beta cells in vivo rather than in vitro, through transgenic manipulation of mice. The transforming agent, SV40 T-antigen (Tag), was expressed under the control of an insulin specific promotor and the tumourous beta cells, denoted $\beta$ TC, were further propagated in culture. Similar cell lines could be derived from other transgenic animals, such as the pig, which may be more appropriate for transplantation into humans. In addition, cultured human islets could be engineered using similar genetic approaches, to derive continuous human beta-cell lines, if the problem of integrating genes into post-mitotic cells was to be solved. The $\beta$ TC cells are highly differentiated and contain about one third of the normal amounts of insulin. They respond well to insulin secretagogues, most importantly to glucose, in the physiological concentration range. The expression of endogenous mouse insulin was maintained, but the issue of non-human vs human insulin is an important one because of immunogenicity and perhaps mitogenicity (see above). Transformed xenografts have raised a number of concerns about safety. To prevent uncontrolled cell growth, the expression of the transforming protein should be tightly regulated. This is also likely to contribute to improved cell function since a reverse correlation exists between proliferation and differentiation. Therefore, a regulatory system was introduced that is based on the bacterial tetracycline (tet) operon to control the expression of the Tag oncoprotein [5]. Using a double-transgenic mouse approach, beta-cell tumours were generated that expressed the $\mathrm{T}$ antigen under the control of a minimal promoter and a reiterated tet-operator. A separate transgene expressed a chimeric transactivator comprising the DNA-binding region of the tet repressor and the transactivating domain of the HSV protein VP16. Tetracycline, as a ligand of the tet-repressor, turns off transactivation of Tag expression. Since the cells maintain an absolute dependence on Tag for their continuous proliferation, they undergo complete growth arrest in the absence of Tag. This on/off regulation can be repeated as needed since it is fully reversible. Thus, this method allows controlled cell expansion both in culture and in vivo. When tet $(1 \mathrm{mg} / \mathrm{ml})$ was added to their drinking water, the incorporation of tritiated thymidine in islets of transgenic mice, as measure for cell proliferation, was dramatically diminished [5]. These results demonstrate that expression of Tag is strictly necessary for proliferation of $\beta$ TC-tet cells, which makes it a secure, tightly regulated system. It should be noted that tetderivatives without antibiotic activity are readily available. The function of these cells, denoted $\beta$ TCtet, is indistinguishable from that of normal beta cells. After 2-3 weeks of growth arrest in culture, their insulin content is increased 3-5 fold, compared with proliferating cells, to the levels of normal islets. Insulin secretion is correctly regulated and is not affected much by prolonged growth arrest in vitro. The regulation of cell growth and function is maintained when the cells are transplanted into streptozotocin-diabetic syngeneic recipients. While control animals were severely hyperglycaemic, transgenics became hypoglycaemic within 30 days. After the administration of a pellet of slowly releasable tet, euglycaemia was maintained during 4-month observation periods in vivo.

As an alternative to xenotransplantation, allotransplantation may succeed without the need for immunoisolation with its attendant unsolved problems. A number of viruses have evolved proteins which are capable of down regulating the host immune response against virus-infected cells. These can be used to render mammalian cells resistant to certain immune responses. One example is human adenoviruses (Ad), which encode a number of such proteins in the early region 3 (E3). One of these, gp19, blocks the 
transport of the class I major histocompatibility (MHC) heavy chain from the endoplasmic reticulum to the cell surface. A group of three other proteins encoded in this region inhibit apoptosis induced by tumour necrosis factor $\alpha(\mathrm{TNF} \alpha)$. To evaluate the potential use of these functions in allogeneic cell transplantation and in the prevention of recurrent autoimmunity, the Ad E3 genes were expressed in pancreatic beta cells of transgenic mice under the control of the insulin promoter (RIP-E3) [6]. Islets from RIPE3 mice transplanted across allogeneic barriers survived for prolonged periods of time (3-4 months), compared with non-transgenic control islets. The effects of the Ad E3 genes on autoimmunity to beta cells were evaluated in the lymphocytic choriomeningitis virus (LCMV) transgenic model. These mice express LCMV proteins in beta cells and develop antiislet autoimmunity and diabetes following infection with the virus. Islets of double transgenic mice co-expressing E3 and LCMV proteins in beta cells became resistant to the virus-induced autoimmunity. Cytotoxic T cells were occasionally found at the periphery of the graft, but the inflammation was never destructive since the $\mathrm{T}$ cells did not recognize their MHC-negative target. These findings indicate that class I MHCmediated antigen recognition, as well as TNF $\alpha$-induced apoptosis, play a role in both allogeneic transplant rejection and autoimmune destruction model for spontaneous autoimmune diabetes.

\section{Clark Colton: Immunoisolation of islet cells}

Dr. Clark Colton presented an overview of the promise and problems of immunoisolation of islet cells [7]. The original concept was that encapsulating donor cells in permselective biodevices would both maintain cell viability and protect from immune destruction by high molecular weight antibodies and lymphocytes. Several configurations have been engineered. However, small immune effector molecules such as cytokines and reactive oxygen species pose a further problem for xenograft tissues. If the membrane is designed so that the tissue graft is freely accessible for circulating growth factors and oxygen, the cut-off will prevent infiltration by cells and circulating antibodies but not by cytokines, free radicals and other small intercellular signalling molecules that mediate the cellular immune response. Problems remaining to be solved include those of capsule volume and retrieval, capsule degradation, and leakage of immunogenic peptides that stimulate immune responses.

\section{Future directions and recommendations}

Among the areas we deem to be most crucial for future research are the following:
- Identification of specific beta-cell precursor markers and the development of techniques for the isolation of these stem cells.

- Elucidation of the genes and their protein products that regulate the growth and differentiation of betacell precursors.

- Identification of genes and their protein products that regulate glucose-stimulated insulin release, including those involved in glucose recognition, stimulus-secretion coupling mechanisms, and exocytic mechanisms.

- Development of a gene-transfer vector that can stably integrate genes into non-dividing differentiated adult human beta cells.

- Identification of genes and gene products that can abrogate allogeneic or xenogeneic rejection processes when expressed in human or animal beta cells, respectively.

- Identification of genes and gene products than can abrogate autoimmune destruction when expressed in human beta cells.

- Identification of genes that can induce post-mitotic human beta cells to proliferate while maintaining chromosomal stability.

- Development of immunoisolation materials that can prevent islet destruction by low molecular weight toxins such as cytokines and reactive oxygen species, while permitting transport of necessary beta-cell growth-promoting factors and oxygen.

\section{References}

1. Beatti GM, Rubin JS, Mally MI, Otonkoski T, Hayek A (1996) Regulation of proliferation and differentiation of human fetal pancreatic islet cells by extracellular matrix, hepatocyte growth factor and cell-cell contact. Diabetes 45: $1223-1228$

2. Otonkoski T, Beatti GM, Mally MI, Ricordi C, Hayek A (1993) Nicotinamide is a potent inducer of endocrine differentiation in cultured human fetal pancreatic cells. J Clin Invst 92: 1459-1466

3. Hohmeier HE, BeltrandelRio H, Clark SA, Henkel-Reiger R, Normington K, Newgard CB (1997) Regulation of insulin secretion from novel engineered insulinoma cell lines. Diabetes 46: 968-977

4. Clark SA, Quaade C, Constandy H et al. (1997) Novel insulinoma cell lines produced by iterative engineering of GLUT-2, glucokinase, and human insulin expression. Diabetes 46: 958-967

5. Efrat S, Fusco-Demaine D, Lemberg H et al. (1995) Conditional transformation of a pancreatic beta cell line derived from transgenic mice expressing a tetracycline-regulated oncogene. Proc Natl Acad Sci USA 92: 3576-3580

6. Efrat S, Fejer G, Brownlee M, et al. (1995) Prolonged survival of pancreatic allografts mediated by adenovirus immunoregulatory transgenes. Proc Natl Acad Sci USA 92: 6947-6951

7. Colton CK (1995) Implantable biohybrid artficial organs. Cell Transplant 4: 415-436 